\title{
Instructions to Authors and Contributors / Directives aux Auteurs
}

\section{General}

Address correspondence or scientific and technical papers to D.M. Burgess; other editorial correspondence should be addressed to V.J. Nordin.

Contributions may be in French or in English. Double space all material intended for The Forestry Chronicle. Three copies will ensure the material will not get lost between editing, production and printing.

Do not use "all caps" in titles or headings. Underline only words that are to appear in italics e.g. scientific names.

Title — titles should be kept short - no more than 10 words

Author documentation - all authors' names and complete mailing address should be included at the bottom of the cover page.

\section{Scientific and Technical and Professional Articles}

There is a charge of $\$ 100$ per page. Page charges may be waived for papers reporting private research or for professional papers prepared privately. Requests for waiving of page charges must be made at the time the paper is submitted. The page charges include one table for each page of article. Additional tables are $\$ 11.00$ each.

Submit the original and two copies of the manuscript, typed doublespaced on white bond paper $8-1 / 2 \times 11$ in. with wide margins; preferably with numbered lines. The first page should contain only the title, the authors, and footnote giving the author(s) affiliation. Footnotes should be numbered consecutively throughout the paper, except in tables. Headings and subheadings should be flush left in upper and lower case letters. References should be listed alphabetically under the heading References and referred to by the author and date, e.g. (McPherson et al. 1982) for:

McPherson, J.A., E.K. Morgenstern and B.S.P. Wang. 1982. Seed production in grafted clonal orchards at Longlac, Ontario. For. Chron. 58(1): 3134.

(a) single-author articles preceed multiple author articles for which the individual is the senior author

(b) two or more articles by the same author(s) are listed chronologically; two or more in the same year are indicated by letters a, b, etc.

(c) all published works in the text should be listed; all listed references should be notated in the text.

(d) material not available through libraries (e.g., personal communication, privileged data) should be cited in the text in parenthesis.

(e) Book references must include author(s), year, title, publisher, city and number of pages (p.) (in that order).

(f) Chapter references from books must include author(s), year, chapter title, In editor(s), book title, pages (pp.), publisher and city (in that order).

(g) Articles, symposium proceedings etc. follow similar reference format.

In review articles or articles where references are of incidental interest, the references may be numbered and referred to in the text by number. Use footnotes for references to unpublished material.

\section{Submission of Manuscripts on Diskette}

Manuscripts may be submitted on 3.5- or 5.25-inch diskettes in IBM Wordperfect. When preparing papers for submission on diskette follow the publication style as closely as possible with regard to headings, capitalization, etc. (see a recent issue of The Forestry Chronicle). Text intended to be set in italic should be underlined, text to be set in bold should be coded bold on Wordperfect; otherwise avoid superfluous word processing codes.

Some specific points:

- Use the numeral 1 (not lower case 1 (ell)) for the number one.

- Use the numeral 0 (not capital O) for zero.

- Do not double space after a period.

- Avoid the use of tabs and indents; be consistent in how you end and start paragraphs.

- Note any special characters used in the document.

- Send a print out of the document as it appears on the disk, not a later or earlier version.

Note: Since all corrections and editing are done on a hard copy of the

\section{Généralités}

Le correspondance sur les articles scientifique professionnels devrait être addressée à M.D.M. Burgess et le reste du courrier, à M.V.J. Nordin.

Les articles peuvent être présentés en anglais ou en français. Le texte est dactylographié à double interligne en respectant les marges. Le matériel soumis doit nous parvenir en trois exemplaires afin d'assurer le suivi entre l'édition, la production et l'impression. Il faut utiliser dans le titre non pas uniquement les majuscules mais les hauts et les bas de case de façon normale comme dans le corps du texte. Seule les mots qui doivent apparaître en italique dans l'épreuve sont soulignés.

Titre - Les titres devraient être le plus court possible, pas plus de 10 mots.

Identification de l'auteur - Le nom de tous les auteurs ainsi que leur adresse postale complète devrait apparaître au bas de la page frontispice.

\section{Articles scientifiques, techniques et professionnels}

Des frais de publication de $100 \$$ par page sont exigibles. Ces frais peuvent être annulés advenant qu'il s'agisse d'articles falsant état de recherche indépendante, ou d'articles professionnels préparés individuellement. La requête pour l'annulation des frais doit accompagner l'article soumis. Les frais de publication comprennent la reproduction d'un tableau par page. Les tableaux supplémentaires exigeront des frais de $11 \$$ par tableau.

L'auteur soumet l'original et deux copies de son manuscrit sur feuille 8$1 / 2 \times 11$ et à lignes numérotées dans la mesure du possible. La première page contient uniquement le titre et les auteurs avec une note en bas de page sur l'appartenance de ceux-ci. Les notes en bas de page rattachées au corps du texte doivent être numérolées consécutivement dans l'article, excepté celles qui accompagnent les tableaux et les figures. Les entêtes et les sousentêtes doivent commencer à la marge avec les caractères de haut et bas de case. La liste des références suit l'ordre alphabétique et la référence dans le texte donne le nom de l'auteur et la date comme suit: pour "Robitaille L. 1977. Recherches sur les feuillus nordiques à la station forestière de Duchesnay. For. Chron. 57: 201-203.", on met (Robitaille 1977).

(a) les articles rédigés par un seul auteur précèdent les articles de plusieurs auteurs pour lesquels l'individu est considéré comme auteur principal

(b) deux ou plusieurs articles rédigés par le ou les mêmes auteurs sont présentés par ordre chronologique; deux ou plusieurs articles rédigés la même année sont identifiés par les lettres $\mathrm{a}, \mathrm{b}$, etc.

(c) tous les travaux publiés cités dans le texte devraient être identifiés dans les références; toutes les références citées devraient être notées dans le texte.

(d) le matériel non disponible en bibliothèque (p.e. communication personnelle, données privilégiées) devrait être cité entre parenthèses.

(e) Les références à des livres doivent inclure le ou les auteurs, l'année, titre, maison d'édition, ville, nombre de pages (p.) (dans cet ordre)

(f) les références à des chapitres tirés de livres doivent inclure le ou les auteurs, le titre du chapitre, in éditeur(s), titre du livre, pages (pp.), maison d'édition et ville. (dans cet ordre)

(g) les articles, les actes de colloques, etc, suivent un format similaire de référence.

On peut cependant, le cas échéant pour des articles constituant une revue de litérature impliquant beaucoup de référence et d'auteurs, procéder par numération dans le texte. Utilisez les notes en bas de page pour les références aux textes inédits.

\section{Soumission de manuscrits sur disquette}

Les manuscrits peuvent être soumis en version Wordperfect de IBM sur des disquettes de 3.5 ou 5.25 pouces. Lors de l'élaboration de l'exposé à soumettre sur une disquette, veuillez suivre les normes de publication aussi fidèlement que possible en ce qui a trait aux titres, majuscules, etc. (Veuillez vous référer à une édition récente du Forestry Chronicle). Le texte qui devrait être en italique, sera souligné, le texte en caractère gras, devrait être encodé en caractère gras selon Wordperfect; en toutes autres circonstances, évitez l'utilisation superflue des codes du traitement de texte.

Quelques points spécifiques: 
manuscript, usually in red pen to be more visible to the author, diskette submissions are not needed until after acceptance, that is, after minor revisions have been completed.

\section{Tables}

Each table should appear on a separate sheet and be numbered in arabic numerals. Only the first word of the title should be capitalized, and similarly only the first word of table headings should be capitalized. Horizontal rules only are used, see The Forestry Chronicle for examples. Tables will appear as one column (3 3/8"), page width (6 5/8") or for large tables sideways on the page (95/8").

\section{Illustrations}

Line illustrations should be in black ink on white paper. Photo-reproductions of line illustrations are acceptable, but photocopies and illustrations produced on dot-matrix printers are not. When planning illustrations bear in mind that they will be reduced to fit the page or column size. All elements of the illustration should be clearly legible when reduced; lettering should be at least $1.5 \mathrm{~mm}$ high when reduced. Non-professional hand or typewritten lettering is not acceptable.

Halftone illustrations (photographs) should be clear black and white prints with good tonal contrast and sharp focus. Previously screened photographs are not acceptable.

All illustrations should be labelled with the first author's name and figure number.

\section{The Abstract}

The abstract is an abbreviated, accurate representation of the paper, of about 50-100 words, rarely longer. The abstract and up to 12 key words or phrases should appear on a separate page. The abstract should be informative. An informative abstract contains the most essential information given in the original. Results and conclusions should be given in numerical values where practical. It may indicate dates, localities, names of species, new methods etc. Information given in the title should not be repeated. The abstract should be able to stand by itself. It should not be used for second thoughts. The abstract is the most important part of a paper because it is read by the most people.

For long reviews or discussions an indicative abstract may be used. It is very short, often only a sentence which expands or clarifies the title.

Abstracts should be submitted in English and in French where possible.

\section{Spelling and Abbreviations}

The Forestry Chronicle uses the Oxford dictionary as its source for the correct spelling of words. Words such as "fertilizer" should be spelled with a " $z$ ", not an "s".

\section{Miscellaneous}

Use Native Trees of Canada for common and scientific names or Checklist of United States Trees by E.L. Little, Jr., Agriculture Handbook No. 541 for a broader list of scientific names. For technical articles the Council of Biology Editors Style Manual (fifth edition) provides useful guidelines on form and style.

Colour photographs related to articles are solicited for possible use on the cover of The Forestry Chronicle.

Submissions not complying with these instructions or not including three copies will be returned or delayed until the required changes have been made.
- Utilisez le caractère numérique 1 (et non le "l" minuscule) pour imprimer le chiffre un.

- Utilisez le caractère numérique 0 (et non le "O" majuscule) pour le zéro.

- N'insérez pas de double espace après un point.

- Evitez l'utilisation de taquets de tabulation et de commande de texte en retrait, soyez consistant dans la façon de débuter et de finir les paragraphes.

- Identifiez tous les caractères spéciaux utilisés dans le document.

- Faites parvenir une copie du document tel qu'il apparaît sur la disquette et non une version antérieure ou plus récente.

N.B. Puisque toutes les corrections et l'édition sont effectuées sur une copie imprimée du manuscrit, habituellement à l'encre rouge afin d'être plus apparentes aux yeux de l'auteur, le dépôt d'une disquette n'est pas nécessaire après son approbation, du moins si les corrections mineures ont été effectuées.

\section{Les tableaux}

Tout tableau doit être présenté sur une feuille séparée et numéroté en chiffre arabe. Seul le premier mot du titre de même que le premier mot d'une case du tableau doit être affecté de la majuscule. La ligne horizontale est la seule en usage dans la revue. Le tableau doit s'accommoder soit de la largeur de la colonne (3-3/8") soit de la largeur de la page (6-5/8") ou encore du plus grand côté de la page (9-5/8").

\section{Illustrations}

Les dessins devraient être réalisés à l'encre noire sur du papier blanc. Les reproductions par procédé photographique des dessins sont acceptables, mais les photocopies et les illustrations produites par une imprimante à points ne le sont pas. Lors de la planification des illustrations, veuillez garder en mémoire qu'elles seront reproduites de façon à s'insérer sur une page ou sur une colonne. Tous les éléments des illustrations devraient être clairement lisibles une fois l'illustration réduite; le corps des lettres devrait être d'une hauteur d'au moins $1.5 \mathrm{~mm}$ une fois réduit. Les lettres à main levée ou à la machine à écrire ne sont pas acceptables.

Les illustrations en demi ton (photographies) devraient prendre la forme d'épreuves claires en noir et blanc ayant un haut niveau de contraste tout en étant très nettes. Des épreuves réalisées à partir d'une projection sur écran ne sont pas acceptables.

Toutes les illustrations devraient être identifiées selon le nom des auteurs et le numéro de l'illustration.

\section{Le résumé}

Le résumé est produit dans la langue de l'article et si possible dans l'autre langue reconnue par la revue. Il donne en abrégé le contenu de l'article en utilisant entre 50 et 100 mots, rarement plus. Cependant, en présence d'un long article de discussion ou de revue de litérature, la résumé peut être réduit à une seule phrase qui vient uniquement clarifier le titre, il peut procéder selon l'ordre de l'article, mais il est plus efficace s'il donne d'abord les éléments les plus importants et les détails de support ensuite. Il est important de se rappeler que le résumé est un document d'information important, lu par le plus grand nombre. Complet par lui-même, il donne l'essentiel de l'article: dates, lieux, noms des espèces et méthodes nouvelles de travail, il évite de répéter ce que donne déjà le titre ou d'ajouter des réflexions. Le résumé doit être présenté sur une feuille séparée accompagné des expressions et mots clés (maximum: 12 ).

\section{Remarques}

L'usage des noms scientifiques doit suivre la nomenclature employée dans "Les arbres indigènes du Canada" ou encore pour les noms anglais celle de "United States Trees" par E.L. Little, Jr., Agriculture Handbook, No 541 . Si le nom de l'espèce n'a pas de correspondant reconnu en français et en anglais, on se limite au nom latin.

L'orthographe des mots anglais doit suivre le dictionaire Oxford pour ce qui est de l'orthographe correcte des mots, français le "Robert". En anglais "fertilizer" doit être écrit avec un "z" et non un "s".

Les normes d'usage sur la forme et le style des articles techniques se retrouvent dans "The Council of Biology Editors Style Manual" ( $5^{\text {ieme }}$ édition).

Les photos en couleurs reliées aux manuscrits sont désirées comme candidats pour le couvert de la revue.

Les articles soumis qui ne se conforment pas à ces instructions ou qui ne comprennent pas trois copies seront retournés à leurs auteurs ou seront reporté tout que les corrections n'auront pas été effectuées. 